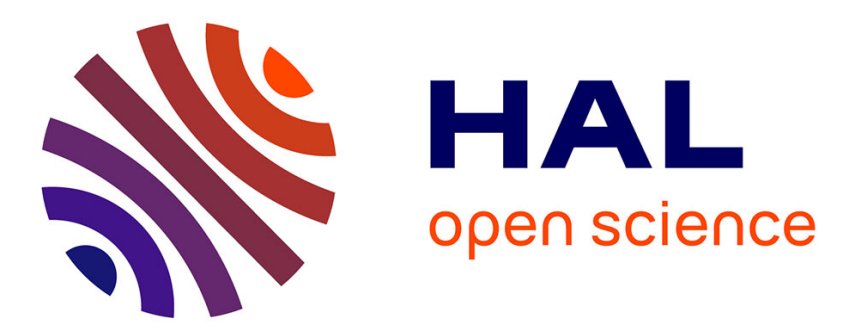

\title{
Slippery but Tough: The Rapid Fracture of Lubricated Frictional Interfaces
}

Elsa Bayart, I. Svetlizky, J. Fineberg

\section{To cite this version:}

Elsa Bayart, I. Svetlizky, J. Fineberg. Slippery but Tough: The Rapid Fracture of Lubricated Frictional Interfaces. Physical Review Letters, 2016, 116 (19), 10.1103/physrevlett.116.194301 . hal03025946

\section{HAL Id: hal-03025946 \\ https://hal.science/hal-03025946}

Submitted on 26 Nov 2020

HAL is a multi-disciplinary open access archive for the deposit and dissemination of scientific research documents, whether they are published or not. The documents may come from teaching and research institutions in France or abroad, or from public or private research centers.
L'archive ouverte pluridisciplinaire $\mathbf{H A L}$, est destinée au dépôt et à la diffusion de documents scientifiques de niveau recherche, publiés ou non, émanant des établissements d'enseignement et de recherche français ou étrangers, des laboratoires publics ou privés. 


\title{
Slippery but Tough: The Rapid Fracture of Lubricated Frictional Interfaces
}

\author{
E. Bayart, I. Svetlizky, and J. Fineberg* \\ The Racah Institute of Physics, The Hebrew University of Jerusalem, Jerusalem 91904, Israel \\ (Received 29 January 2016; revised manuscript received 23 March 2016; published 10 May 2016)
}

\begin{abstract}
We study the onset of friction for rough contacting blocks whose interface is coated with a thin lubrication layer. High speed measurements of the real contact area and stress fields near the interface reveal that propagating shear cracks mediate lubricated frictional motion. While lubricants reduce interface resistances, surprisingly they significantly increase the energy dissipated $\Gamma$ during rupture. Moreover, lubricant viscosity affects the onset of friction but has no effect on $\Gamma$. Fracture mechanics provide a new way to view the otherwise hidden complex dynamics of the lubrication layer.
\end{abstract}

DOI: 10.1103/PhysRevLett.116.194301

The lubrication of solid surfaces is generally used to reduce frictional resistance to sliding motion and to prevent material wear [1]. The effects of fluids on the frictional properties of an interface are of particular significance in geophysics, since tectonic faults are generally lubricated by interstitial water or melted rocks [2-5]. Along spatially extended multicontact interfaces, which are considered here, much of the fundamental understanding of the collective mechanisms responsible for the reduction of friction due to lubrication is still lacking [6-8]. While the sliding dynamics of lubricated systems is an active field of research [9-11], the mechanisms mediating their transition from stick to slip remain largely unexplored. At the microscopic level, stickslip mechanisms have been discussed for decades $[12,13]$. Within single contacts, thin films, typically at a nanometric size, exhibit enhanced strength but the responsible mechanisms remain unclear [14-18].

Along spatially extended rough interfaces, the real contact area is defined by a large ensemble of single contacts (asperities) that couple contacting elastic blocks. The real contact area $A$ is generally orders of magnitude smaller than the apparent one $[1,19,20]$. Here, we consider rough surfaces in the boundary lubrication regime, where the contacting surfaces are covered by a thin lubricant layer [21]. The discrete asperities in this regime still bear the entire normal load; they are not entirely immersed in the fluid layer as in the full lubrication regime. The mixed lubrication regime is an intermediate region, where the normal load is partially borne by solid contacts and partially by the liquid layer.

In dry friction, the onset of motion is mediated by rupture fronts propagating along the frictional interface [22,23]. These fronts are true singular shear cracks; the strain fields during their propagation are well described by linear elastic fracture mechanics (LEFM) [24,25]. Frictional rupture arrest is also governed by the same framework [26,27].

Here, we examine the mechanisms coming into play when motion initiates within lubricated interfaces, in the boundary lubrication regime. We first find that interface rupture still corresponds to the shear cracks described by LEFM. While reducing static friction by facilitating rupture nucleation, we will show that, surprisingly, lubricants make solid contacts effectively tougher, increasing the fracture energy of the interface (the dissipated energy per unit crack extension). Moreover, while the macroscopic frictional resistance of the interface depends on the lubricant viscosity, the fracture energy does not. We use this to demonstrate that nucleation and propagation of frictional ruptures are independent processes.

We describe experiments where two blocks of poly (methylmethacrylate) (PMMA) are first pressed together with normal forces $F_{N}$ of $2500<F_{N}<7000 \mathrm{~N}$. Shear forces $F_{S}$ are then applied uniformly, as the bottom block is translated via a rigid stage, until stick-slip motion initiates (Fig. 1). A detailed description of the setup is given in Ref. [24]. PMMA has a rate-dependent Young's modulus of $3<E<5.6 \mathrm{GPa}$ and a Poisson ratio $\nu_{p}=0.33$. PMMA's Rayleigh wave speed is $c_{R}=1255 \mathrm{~ms}^{-1}$ for plane strain
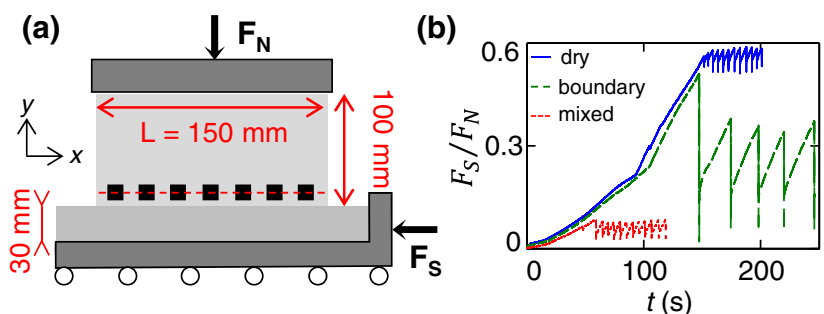

FIG. 1. Experimental setup and stick-slip behavior. (a) Normal $F_{N}$ and shear $F_{S}$ forces are applied to contacting PMMA blocks. Shear is applied uniformly via translation of a rigid stage. Strain gage rosettes measure the three components of the 2D-strain tensor at 14 locations along and $3.5 \mathrm{~mm}$ above the interface, while the real contact area is measured optically. (b) Loading curves, $F_{S} / F_{N}$ versus time, are plotted for typical experiments, with $F_{N}=4000 \mathrm{~N}$ : dry (solid blue line), boundary lubricated (dashed green line), and, for comparison, in the mixed lubricated regime (dotted red line). The lubricant used is a hydrocarbon oil (TKO-77). 
conditions. The top and bottom blocks have respective $x \times y \times z$ dimensions of $150 \times 100 \times 5.5$ and $200 \times 30 \times 30 \mathrm{~mm}$. The contacting flat surfaces of the top and bottom blocks were (top) optically smooth and (bottom) had a surface roughness of $0.5 \mu \mathrm{m} \mathrm{rms}$. The experiments were all performed with the same two blocks, to negate any effects due to surface preparation or roughness. During each sliding event, an array of 14 strain gages recorded the three components of the 2D-strain tensor $\varepsilon_{i j} 3.5 \mathrm{~mm}$ above the interface, each at $10^{6}$ samples/s. The corresponding stresses $\sigma_{i j}$ are calculated from $\varepsilon_{i j}$ after accounting for the viscoelasticity of PMMA (see Ref. [27]). In parallel, the real area of contact $A(x, t)$ was measured at $1000 \times 8$ locations at 580000 frames/s, using an optical method based on total internal reflection (see Ref. [24]) where incident light only traverses the interface at contacts, and is otherwise reflected.

The experiments of lubricated friction were performed using silicone oils with kinematic viscosities of $\nu \sim 5$, 100 , and $10^{4} \mathrm{~mm}^{2} \mathrm{~s}^{-1}$ and a hydrocarbon oil (TKO-77, Kurt J. Lesker Company) with kinematic viscosity of $\nu \sim 200 \mathrm{~mm}^{2} \mathrm{~s}^{-1}$. Lubricants were applied to either or both of the contacting surfaces and then wiped. Our results are not appreciably affected by the wiping procedure (number of wipes, application or not between experiments) or by the cleaning (soap, water, and isopropanol). PMMA and the lubricants used are nearly index matched: PMMA (1.49), TKO-77 (1.48), and silicone oils (1.42). Hence, under total internal reflection, the incident light will be totally transmitted where gaps between asperities are filled with liquid. As light could be transmitted via capillary bridges across contacting surfaces, we only consider relative variations in light intensity. At the onset of motion, the observed contact area variations (see below) demonstrate that air, not lubricant, fills the gaps between contacts. This provides validation that the experiments take place in the boundary lubrication regime.

When sheared, the lubricated system undergoes stickslip motion [Fig. 1(b)]. Drops of $F_{S}$ in the loading curves correspond to slip events with a macroscopic relative displacement of the blocks. The lubricant layer affects the macroscopic frictional resistance, reducing the static friction coefficient (i.e., the shear force threshold). The amplitudes of the force drops, however, are larger than for dry friction. In the boundary lubrication regime, this pattern is extremely robust, and is independent of the nature and quantity of the lubricant. For completeness, a typical loading curve in the mixed lubrication regime is included in Fig. 1(b), where the $F_{S} / F_{N}$ thresholds are further reduced. Motion in this regime is not addressed here.

As in dry friction, each sliding event in the boundary lubrication regime is preceded by propagating rupture fronts that break the solid contacts forming the interface, as shown in Fig. 2(a). Macroscopic sliding only occurs when a front traverses the entire interface [27-30]. For steady rupture fronts moving at a velocity $c_{f}$, $\varepsilon_{i j}(x, t)=\varepsilon_{i j}\left(x-c_{f} t\right)$. Using this and the optically identified location of the rupture tip $x_{\text {tip }}(t)$, we converted $\varepsilon_{i j}(x, t)$ to spatial measurements $\varepsilon_{i j}\left(x-x_{\text {tip }}\right)$ [24]. As in the example of Fig. 2(b) (blue line), the rupture fronts in dry friction are shear cracks whose stress field variations $\Delta \sigma_{i j}(r, \theta)$ are quantitatively described by LEFM, with respect to the crack tip $(r=0)$ [24]:

$$
\Delta \sigma_{i j}(r, \theta)=\frac{K_{\mathrm{II}}\left(c_{f}\right)}{\sqrt{2 \pi r}} \Sigma_{i j}^{\mathrm{II}}\left(\theta, c_{f}\right),
$$

where $\Sigma_{i j}^{\mathrm{II}}\left(\theta, c_{f}\right)$ is a universal angular function and the coefficient $K_{\mathrm{II}}\left(c_{f}\right)$, is called the stress intensity factor [31]. $\Delta \sigma_{i j}$ expresses the stress changes between the initially applied and residual stresses along the frictional crack faces. The $\Delta \sigma_{i j}$ are related to the measured strain variations $\Delta \varepsilon_{i j}$ via the dynamic Young's modulus and Poisson ratio of PMMA. LEFM relates $K_{\text {II }}$ to the fracture energy $\Gamma$, the energy dissipated per unit crack advance; $K_{\mathrm{II}} \propto f\left(c_{f}\right) \sqrt{\Gamma}$, where $f\left(c_{f}\right)$ is a known universal function [31].

In Fig. 2(b) we compare measurements of $\Delta \varepsilon_{i j}\left(x-x_{\text {tip }}\right)$ during the rupture front propagation for dry and lubricated interfaces. Following Eq. (1), fitting the three strain
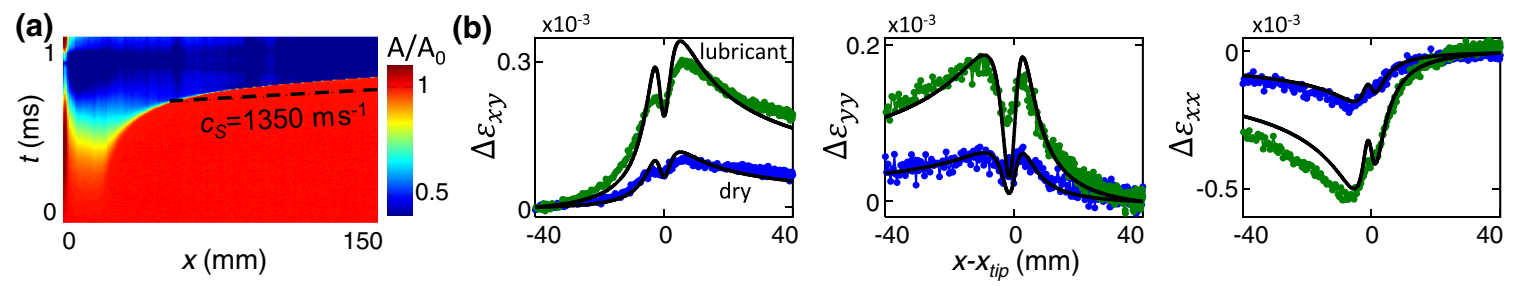

FIG. 2. Singular interfacial shear cracks govern friction initiation. (a) The spatiotemporal evolution of the contact area $A(x, t)$ of a typical lubricated interface (hydrocarbon oil, TKO-77). Each line is a snapshot in time of $A(x, t)$, normalized by $A_{0}=A(x, 0)$ immediately prior to the event. Here, a rupture accelerates to a propagation velocity $c_{f}=0.92 c_{R}$. The rupture tips $x_{\text {tip }}(t)$ are the locations where $A(x, t)$ drops sharply. (b) Variation of the strain field $\Delta \varepsilon_{i j}\left(x-x_{\text {tip }}\right)$ with the distance from the rupture tips $x_{\text {tip }}$, for ruptures propagating along dry (blue line) and lubricated [green line, rupture presented in (a)] interfaces. In both, the applied normal stress was $\left\langle\sigma_{y y}\right\rangle=7 \pm 0.5 \mathrm{MPa}$ and strains were measured at $x=77 \mathrm{~mm}$, where $c_{f} \sim 0.3 c_{R}$. Black solid lines are fits to the LEFM solution for $y=3.5 \mathrm{~mm}$ [Eq. (1)]. The only fitting parameter is the fracture energy; $\Gamma_{\text {dry }}=2.6 \pm 0.3 \mathrm{~J} \mathrm{~m}{ }^{-2}$ for the dry and $\Gamma_{\text {lub }}=23 \pm 3 \mathrm{~J} \mathrm{~m}^{-2}$ for the lubricated interfaces. 
components provides a dynamic measurement of $K_{\text {II }}[24,31]$ and, therefore, a measurement of $\Gamma$. As Fig. 2(b) explicitly shows, the agreement between the measured $\Delta \varepsilon_{i j}\left(x-x_{\text {tip }}\right)$ for the lubricated interface and the LEFM solution is excellent. Hence, the ruptures propagating along a lubricated interface are shear cracks. Surprisingly, $\Gamma$, for the same applied normal load, is an order of magnitude greater for the lubricated interface, $\Gamma_{\text {lub }}$, than for the dry one, $\Gamma_{\text {dry }}$. For the examples presented in Fig. 2(b), $\Gamma_{\text {lub }}=23 \pm 3 \mathrm{~J} \mathrm{~m}^{-2}$ while $\Gamma_{\text {dry }}=2.6 \pm 0.3 \mathrm{~J} \mathrm{~m}^{-2}$. In Fig. 3(a) we present $\Delta \varepsilon_{i j}\left(x-x_{\text {tip }}\right)$ for dry and lubricated (hydrocarbon) interfaces, when rescaled by $1 / \sqrt{\Gamma}$. We find that the rescaled dry and lubricated strain fields are indeed identical.

What determines $\Gamma$ ? In dry friction, when the contacts are plastically deformed, $\Gamma$ grows linearly with the normal load $[1,27]$. Extracting $\Gamma$ from the rescaling procedure, Fig. 3(b) shows that $\Gamma$ indeed remains proportional to the average normal stress $\left\langle\sigma_{y y}\right\rangle$ in the boundary lubrication regime. Moreover, the value of $\Gamma$ is unaffected by the lubricant viscosity; $\Gamma$ is constant for viscosity variations of $5<\nu<10^{4} \mathrm{~mm}^{2} \mathrm{~s}^{-1}$ in silicon oils. We do, however, find that $\Gamma$ strongly depends on the lubricant composition; TKO-77 has values of $\Gamma$ about 3 times larger than in all of the silicon oils used. For a given $c_{f}$, increased values of $\Gamma$ induce increased shear stress drops during rupture propagation. The increased shear force drops in loading curves [e.g., Fig. 1(b)] are partially caused by this large stress drop, with the remainder due to motion after the rupture passage.

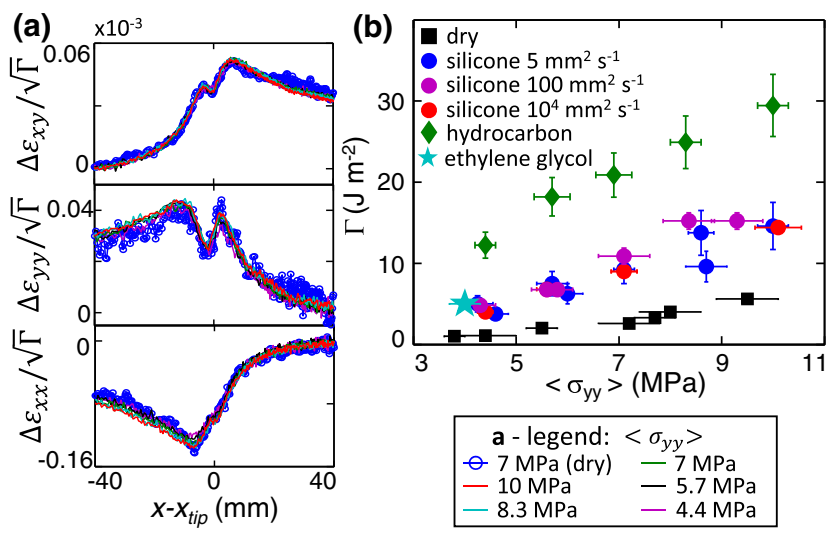

FIG. 3. Dependence of the fracture energy with normal stress. (a) Comparison of $\Delta \varepsilon_{i j}\left(x-x_{\text {tip }}\right)$ for dry and lubricated experiments, when normalized by $\sqrt{\Gamma}$, for different normal loads. Units are $(\mathrm{Pam})^{-1 / 2}$. Superimposed are the dry experiment in Fig. 2(b) and five lubricated (TKO-77) experiments where $c_{f} \sim 0.3 c_{R}$ with $\left\langle\sigma_{y y}\right\rangle$ as in the legend, yielding $\Gamma_{\text {dry }}=2.6 \mathrm{~J} \mathrm{~m}^{-2}$ and $\Gamma_{\mathrm{TKO}}=12.3$, $18.2,23,25$, and $29.5 \mathrm{~J} \mathrm{~m}^{-2}$. (b) $\Gamma$ is measured by fitting the strain field with the LEFM solution [as in Fig. 2(b)] for both the dry and lubricated interfaces versus $F_{N}$. All $\Gamma$ vary linearly with $F_{N} ; \Gamma$ is independent of the lubricant viscosity while being highly dependent on lubricant composition.
Why does the lubricant increase $\Gamma$ ? We consider the simplest (linear slip-weakening) description of the dissipative zone near a rupture tip [32]. In this model, rupture occurs when the shear stress on the interface reaches a maximal value $\sigma_{x y}^{\text {peak }}$. Slip is then initiated and $\sigma_{x y}$ is reduced to the residual value $\sigma_{x y}^{\text {res }}$ over a slip distance $d_{c}$. While simple, this model contains the main features of the regularized dissipative zone, and $d_{c}$ provides an accurate estimate of the sliding distance, typically the asperity size. The fracture energy is expressed as $\Gamma=\frac{1}{2}\left(\sigma_{x y}^{\text {peak }}-\sigma_{x y}^{\text {res }}\right) d_{c}$. More sliding occurs after the rupture passage, dissipating more energy. Therefore, the energy dissipated by the rupture is only part of the total energy dissipated during a slip event. An increase of $\Gamma$ can be induced by increased values of either $\sigma_{x y}^{\text {peak }}$ or $d_{c}$, or a decrease of $\sigma_{x y}^{\text {res }}$.

As Fig. 4(a) shows, $\sigma_{x y}^{\text {res }}$ is indeed strongly reduced by the lubricant. The magnitude of the reduction relative to the dry interface depends on the nature of the lubricant. It is greater

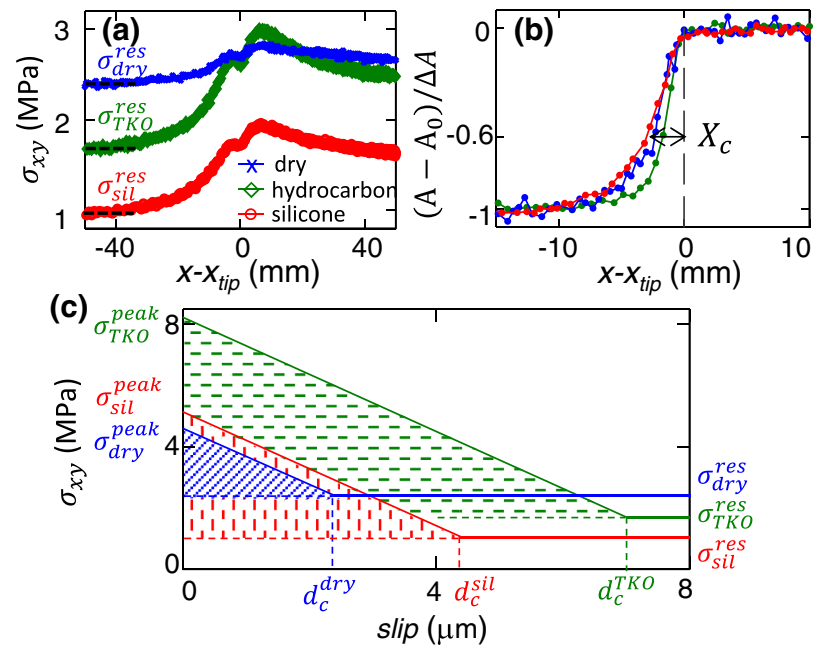

FIG. 4. Measurements of $\sigma_{x y}^{\text {res }}, X_{c}, \sigma_{x y}^{\text {peak }}$, and $d_{c}$. (a) Shear stress as a function of the distance from $x_{\text {tip }}$ for ruptures propagating $\left(c_{f} \sim 0.3 c_{R}\right)$ along a dry (blue crosses) and lubricated interfaces with hydrocarbon oil (green diamonds) and silicone oil with $\nu=10^{4} \mathrm{~mm}^{2} \mathrm{~s}^{-1}$ (red circles). $\Gamma=2.6,9$, and $23 \mathrm{~J} \mathrm{~m}^{-2}$ for, respectively, dry, silicone, and hydrocarbon oils for $\left\langle\sigma_{y y}\right\rangle=7 \mathrm{MPa}$. The blue and green plots are measurements presented in Fig. 2(b), where strain variations are, instead, presented in terms of the absolute stress values. (b) Reduction of the contact area $A-A_{0}$, normalized by the total drop in $A$, $\Delta A=A_{0}-A_{\text {res }}$, as a function of the distance from $x_{\text {tip }}$ for the three experiments in (a). The dissipative zone size $X_{c}$ is defined as the length scale where a $60 \%$ drop of $\Delta A$ occurs. (c) Shear stress versus slip distance where $\sigma_{x y}^{\text {peak }}$ and $d_{c}$ are estimated within the linear slip-weakening model [32]. Respectively, for the dry and lubricated with silicone and hydrocarbon oil interfaces, the residual stresses, defined in (a), are $2.4,1$, and $1.7 \mathrm{MPa}$, the peak stresses $\sigma_{x y}^{\text {peak }}$, using Eq. (2), are 4.6, 5.1, and 8.2 MPa, and $d_{c}$ are $2.4,4.4$, and $7 \mu \mathrm{m}$. Integration over the blue (green, red) hatched areas provides the dry (lubricated) fracture energy. 
for silicone oil than for TKO-77. We cannot measure $\sigma_{x y}^{\text {peak }}$ directly, as our strain gages are located above the interface [24]. The linear slip weakening model provides us with a way to accurately estimate $\sigma_{x y}^{\text {peak }}$ by measuring the size of the dissipative zone $X_{c}$, the distance behind the crack tip over which contacts are being broken [32]:

$$
\sigma_{x y}^{\text {peak }}=\sigma_{x y}^{\mathrm{res}}+\sqrt{\frac{9 \pi}{32} \frac{\Gamma E}{\left(1-\nu_{p}^{2}\right) X_{c}}} .
$$

$X_{c}$ is the scale over which $A(x)$ drops from its initial to its residual value. In Fig. 4(b) we compare $X_{c}$ for the dry and lubricated experiments (see Ref. [24] for details). We find that $X_{c}$ is not significantly affected by the lubricant layer; its value (for $c_{f} \sim 0.3 c_{R}$ ) is approximately $3 \mathrm{~mm}$. Inserting this value in Eq. (2), we see that $\sigma_{x y}^{\text {peak }}$ is not reduced by the lubricant and is even significantly increased when TKO-77 is used [Fig. 4(c)]. The dynamic measurements of the stress drop $\sigma_{x y}^{\text {peak }}-\sigma_{x y}^{\text {res }}$ coupled with $\Gamma$ yield a quantitative estimate of $d_{c}$. Measurements of $\sigma_{x y}^{\text {peak }}, \sigma_{x y}^{\text {res }}, \Gamma$, and $d_{c}$ are presented Fig. 4(c). These measurements indicate that increases in $\Gamma$ are therefore explained by increased stress drops, coupled to larger slip distances.

We have seen that, in the boundary lubrication regime, while the contacts become tougher, requiring a larger amount of energy $(\Gamma)$ to break (Fig. 3), the macroscopic frictional resistance is actually reduced [Fig. 1(b)]. These intriguing results are not contradictory; we show here that rupture nucleation and dissipation are independent processes.

Rupture nucleation determines the initial stress levels and therefore, an interface's "static" frictional strength. Hence, nucleation is the key in understanding the initial interfacial strength, although processes determining how and at what stress levels nucleation takes place remain enigmatic [33,34]. Figure 5 demonstrates that the lubricant viscosity directly affects the initially imposed stress $\sigma_{x y}^{\mathrm{pre}}$ needed to nucleate the rupture. Using silicone oils of different viscosities, in experiments performed with the same normal stress profile, Fig. 5 reveals that the higher $\nu$ is, the lower $\sigma_{x y}^{\text {pre }}$ is. On the other hand, $\sigma_{x y}^{\text {res }}$ does not depend on $\nu . \sigma_{x y}^{\mathrm{pre}}$ determines the static friction coefficient $\mu_{s}=F_{S} / F_{N}$. Hence, as Fig. 5(c) shows, $\mu_{s}$ is significantly dependent on $\nu$, as suggested by earlier studies $[1,35]$.

As $\Gamma$ and $\sigma_{x y}^{\text {res }}$ are $\nu$ independent [Figs. 3 and 5(b)], LEFM predicts that the only effect of $\sigma_{x y}^{\text {pre }}$ should be on the rupture dynamics. A larger $\sigma_{x y}^{\text {pre }}$ yields faster rupture fronts [31] as verified in both dry friction [36] and in ice quakes [37]. This is born out by the examples shown in Fig. 5(d); for the same $\sigma_{y y}$ profile, the higher $\nu$ is, the lower $\sigma_{x y}^{\text {pre }}$ is, and the slower the rupture front is. Our results imply that the reduction of $\mu_{s}$ is purely the result of rupture front nucleation at a reduced threshold, due to a higher $\nu$.

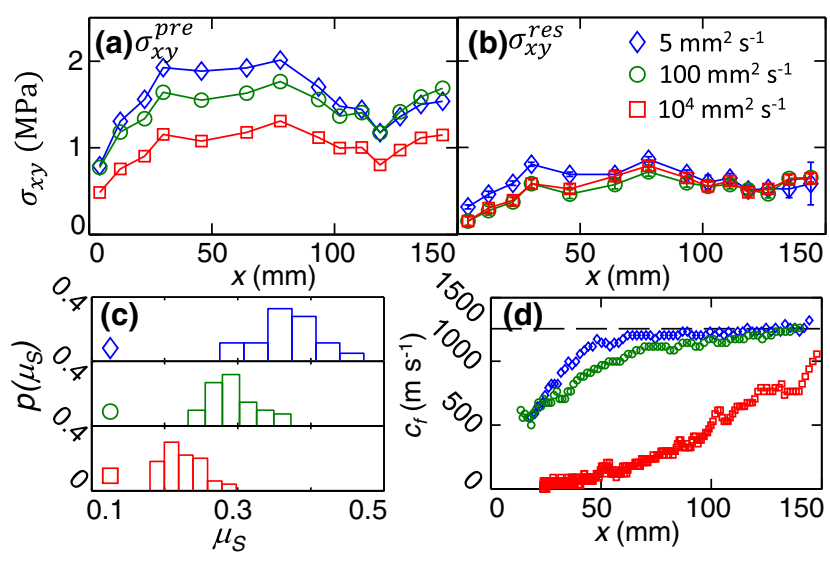

FIG. 5. Dependence of the macroscopic frictional resistance with $\nu$. Profiles of the (a) initial $\sigma_{x y}^{\text {pre }}$ and (b) residual $\sigma_{x y}^{\text {res }}$ stresses for interfaces lubricated with silicone oils having $\nu=5$ (diamonds), 100 (circles), and $10^{4}$ (squares) $\mathrm{mm}^{2} \mathrm{~s}^{-1}$. Normal stress distributions are identical for the three experiments and $\Gamma=6.7 \pm 0.3 \mathrm{~J} \mathrm{~m}^{-2} . \sigma_{x y}^{\text {res }}$ does not depend on the viscosity of the silicone oil. (c) Histograms of the static friction coefficient $\mu_{S}$ of sliding events for lubricated interfaces with silicone oils of different viscosity. Around 50 events are considered for each $\nu$. Higher $\nu$ yield lower frictional resistance. (d) Three examples of $c_{f}(x)$ for the lubricants in (a) and (b). The larger $\nu$ is, the slower the front is. Dashed line denotes $c_{R}$. Same symbols and colors in (a)-(d) as in (b).

Lower initial stresses do not prevent interfacial rupture propagation as long as the elastic energy stored by block deformation is sufficiently above the energy dissipated during the rupture process [Fig. 4(c)].

We have shown that, in the boundary lubrication regime, the interfacial resistance is reduced due to facilitated nucleation of the rupture front (Fig. 5), while the contacts become tougher (Fig. 3). The increase of $\Gamma$ is explained by a strengthening of the contacts (increased $\sigma_{x y}^{\text {peak }}$ ) coupled to the reduced $\sigma_{x y}^{\text {res }}$, with resultant increases in slip distances $d_{c}$. The reduced $\sigma_{x y}^{\text {res }}$ and larger $d_{c}$ may result from the fact that, while in motion, the lubricant layer facilitates slip. The increased values of $\Gamma$ and $\sigma_{x y}^{\text {peak }}$, however, are both new and intriguing observations. In our experiments, on a rough multicontact interface, pressures at a single contact reach the yield stress of PMMA ( 500 MPa) [20], suggesting that nanometric liquid films could well be trapped between asperities. At these extreme conditions the contribution of capillary bridges is negligible to both the frictional resistance and $\Gamma$ (see the Supplemental Material [38]). At a microscopic level, the physics of lubricated single contact interfaces is both interesting and puzzling. Experiments reveal that fluid lubrication layers confined to nanometric scales can transition to solids [15-17]. Other recent experiments on similar systems suggest that the nature of the fluid layers does not change, but a high system stiffness results from the coupling of the fluid to the elastic deformation of the surrounding medium [18]. While our rough, 
multicontact system is far from these ideal cases, it is interesting that lubricant strengthening indeed takes place. Obtaining a fundamental understanding of the dynamics of the lubrication layer and its associated dissipative properties in such a disordered system is an important and interesting challenge.

We acknowledge support from the James S. McDonnell Fund (Grant No. 220020221), the European Research Council (Grant No. 267256), and the Israel Science Foundation (Grants No. 76/11 and No. 1523/15). E. B. acknowledges support from the Lady Davis Trust. We thank G. Cohen for comments.

*jay@mail.huji.ac.il

[1] F. P. Bowden and D. Tabor, The Friction and Lubrication of Solids, 2nd ed. (Oxford University Press, New York, 2001).

[2] J. R. Rice, J. Geophys. Res.: Solid Earth 111, B05311 (2006).

[3] H. Noda, E. M. Dunham, and J. R. Rice, J. Geophys. Res.: Solid Earth 114, B07302 (2009).

[4] N. Brantut and J. R. Rice, Geophys. Res. Lett. 38, L24314 (2011).

[5] R. C. Viesca and D. I. Garagash, Nat. Geosci. 8, 875 (2015).

[6] D. A. Rigney and S. Karthikeyan, Tribol. Lett. 39, 3 (2010).

[7] L. Pastewka, S. Moser, P. Gumbsch, and M. Moseler, Nat. Mater. 10, 34 (2011).

[8] N. N. Gosvami, J. A. Bares, F. Mangolini, A. R. Konicek, D. G. Yablon, and R. W. Carpick, Science 348, 102 (2015).

[9] B. N. J. Persson, Sliding Friction Physical Principles and Applications, 2nd ed. (Springer-Verlag, New York, 2000).

[10] S. Campen, J. Green, G. Lamb, D. Atkinson, and H. Spikes, Tribol. Lett. 48, 237 (2012).

[11] A. Ernesto, D. Mazuyer, and J. Cayer-Barrioz, Tribol. Lett. 59, 23 (2015).

[12] P. A. Thompson and M. O. Robbins, Science 250, 792 (1990).

[13] I. Rosenhek-Goldian, N. Kampf, A. Yeredor, and J. Klein, Proc. Natl. Acad. Sci. U.S.A. 112, 7117 (2015).

[14] D. Y. C. Chan and R. G. Horn, J. Chem. Phys. 83, 5311 (1985).

[15] S. Granick, Science 253, 1374 (1991).

[16] J. Klein and E. Kumacheva, Science 269, 816 (1995).

[17] B. Bhushan, J. N. Israelachvili, and U. Landman, Nature (London) 374, 607 (1995).
[18] R. Villey, E. Martinot, C. Cottin-Bizonne, M. PhanerGoutorbe, L. Leger, F. Restagno, and E. Charlaix, Phys. Rev. Lett. 111, 215701 (2013).

[19] J. Greenwood and J. Williams, Proc. R. Soc. A 295, 300 (1966).

[20] J. H. Dieterich and B. D. Kilgore, Tectonophysics 256, 219 (1996).

[21] B. Hamrock, S. Schmid, and B. Jacobson, Fundamentals of Fluid Film Lubrication (CRC Press, Cleveland, 2004).

[22] S. M. Rubinstein, G. Cohen, and J. Fineberg, Nature (London) 430, 1005 (2004).

[23] K. Xia, A. J. Rosakis, and H. Kanamori, Science 303, 1859 (2004).

[24] I. Svetlizky and J. Fineberg, Nature (London) 509, 205 (2014).

[25] I. Svetlizky, D. Pino Munoz, M. Radiguet, D. S. Kammer, J.-F. Molinari, and J. Fineberg, Proc. Natl. Acad. Sci. U.S.A. 113, 542 (2016).

[26] D. S. Kammer, M. Radiguet, J. P. Ampuero, and J. F. Molinari, Tribol. Lett. 57, 23 (2015).

[27] E. Bayart, I. Svetlizky, and J. Fineberg, Nat. Phys. 12, 166 (2016).

[28] S. M. Rubinstein, G. Cohen, and J. Fineberg, Phys. Rev. Lett. 98, 226103 (2007).

[29] O. M. Braun, I. Barel, and M. Urbakh, Phys. Rev. Lett. 103, 194301 (2009).

[30] J. Tromborg, J. Scheibert, D. S. Amundsen, K. Thogersen, and A. Malthe-Sorenssen, Phys. Rev. Lett. 107, 074301 (2011).

[31] L. B. Freund, Dynamic Fracture Mechanics (Cambridge, New York, 1990).

[32] A. C. Palmer and J. R. Rice, Proc. R. Soc. A 332, 527 (1973).

[33] Z. P. Yang, H. P. Zhang, and M. Marder, Proc. Natl. Acad. Sci. U.S.A. 105, 13264 (2008).

[34] S. Latour, A. Schubnel, S. Nielsen, R. Madariaga, and S. Vinciguerra, Geophys. Res. Lett. 40, 5064 (2013).

[35] W. Hardy, Collected Works (Cambridge University Press, Cambridge, England, 1936).

[36] O. Ben-David, G. Cohen, and J. Fineberg, Science 330, 211 (2010).

[37] J. I. Walter, I. Svetlizky, J. Fineberg, E. E. Brodsky, S. Tulaczyk, C. G. Barcheck, and S. P. Carter, Earth Planet. Sci. Lett. 411, 112 (2015).

[38] See Supplemental Material at http://link.aps.org/ supplemental/10.1103/PhysRevLett.116.194301 for an estimation of the capillary bridges contribution to fracture energy and contact strength. 